\title{
Características dos concursos para contratação de cirurgiões-dentistas na Estratégia Saúde da Família
}

\author{
Leonardo Ribeiro da Silva Moço*; Flávia Martão Flório**; Luciane Zanin**
}

* Mestre em Odontologia, área de Saúde Coletiva, Faculdade São Leopoldo Mandic, Campinas

** Docente, Departamento de Saúde Coletiva, Curso de Odontologia, Faculdade São Leopoldo Mandic, Campinas

Recebido em 19/01/2017. Aprovado em 20/04/2017.

\begin{abstract}
RESUMO
O objetivo do presente estudo foi avaliar as características dos concursos públicos para contratação de cirurgiões-dentistas para a Estratégia Saúde da Família em municípios Brasileiros. Foi realizada análise documental de editais de concursos públicos ocorridos entre 2006 e 2014 no Brasil, com base nos seguintes critérios: macrorregião, conteúdo programático, salário, número de vagas e regime de contratação. De um total 765 editais de concursos, $42 \%$ eram para a região Sudeste, $24 \%$ Nordeste, $22 \%$ Sul, $7 \%$ Centro-Oeste e 5\% Norte. A média de vagas ofertadas foi de 4,2 e de salário foi de R \$ $2.458,01$. Os conteúdos foram distribuídos em conhecimento específico (81\%), língua portuguesa (71\%), conhecimentos gerais $(30 \%)$, saúde coletiva (14\%) e outros $(33 \%)$. Com relação às formas de contratação, $21 \%$ estavam relacionadas a regime de trabalho estatutário, $16 \%$ celetista, $4 \%$ processo seletivo de caráter temporário, $1 \%$ contrato temporário na ausência de processo seletivo público e $57 \%$ não especificaram. Os concursos públicos no período avaliado mostraram editais imprecisos, com ausência de informações importantes para a decisão segura em participar do processo seletivo. A maioria dos concursos foi realizada na região Sudeste, estado de Minas Gerais, com a exigência de conhecimentos específicos da área de atuação e língua portuguesa. Conteúdos de Saúde Coletiva e titulação foram exigidos apenas pela minoria. Faz-se necessário o direcionamento, principalmente em relação aos conhecimentos exigidos nas provas, para que seja contemplado o profissional com perfil e conhecimentos adequados para o trabalho na Estratégia de Saúde da Família.
\end{abstract}

Descritores: Odontólogos. Seleção de Pessoal. Sistema Único de Saúde. Saúde da Família.

\section{INTRODUÇÃO}

O processo de formação acadêmica dos profissionais de saúde e, dentre estes, os de
Odontologia, é influenciado por vários fatores externos às instituições de ensino, incluindo as mudanças de natureza social, econômica, cultural 
e tecnológica, bem como as relacionadas às políticas de educação e de saúde ${ }^{1}$.

No Brasil, a Atenção Primária a Saúde (APS) ganha cada vez mais notoriedade e é alçada como prioridade do Sistema Único de Saúde (SUS), sobretudo, após 1994 com a implantação do Programa Saúde da Família (PSF), cujo objetivo foi reorganizar os serviços e práticas assistenciais e reordenar a demanda, efetivando-se como porta de entrada do sistema. Entretanto, a implementação e consolidação do PSF, que anos depois passou a ser considerado como Estratégia Saúde da Família (ESF), sofreram dificuldades ${ }^{2,3}$ dentre as quais observase a existência de nós críticos na contratação e capacitação de recursos humanos, na fixação e na integração entre os profissionais de saúde.

Com a inclusão da saúde bucal na ESF, em $2001^{4}$ ampliou-se as perspectivas de mercado de trabalho para o cirurgião-dentista (CD), despontando o serviço público como alternativa promissora $^{5}$. Além disso, diante de todas as inovações nos conceitos e no desenho da atenção em saúde, os CDs necessitam de conhecimentos e habilidades adicionais, ou seja, uma formação comprometida com a Saúde Pública/Saúde Coletiva para que possam atuar nos moldes desta estratégia, que se encontra em expansão ${ }^{6}$.

Vem sendo discutida a necessidade de reorientar a formação acadêmica dos CDs, uma vez que estes são precocemente direcionados para as especialidades ${ }^{7}$, não sendo efetivamente preparados para o mercado de trabalho atual que busca a formação de um profissional generalista.

Neste sentido, as instituições responsáveis pela formação de profissionais de saúde devem incorporar na formação do profissional condições que os habilitem a trabalhar em nível comunitário, buscando resgatar a cidadania dos indivíduos, com vistas à construção de um modelo de atenção que contemple a integralidade, a dignidade e a ética ${ }^{6}$ com um perfil generalista com sólida formação técnico- científica, humanística e ética, orientada para a promoção de saúde, com ênfase na prevenção de doenças bucais prevalentes ${ }^{8}$. O perfil dos profissionais de saúde bucal necessita de uma adequação para atender aos princípios do SUS e da ESF, visto que a maioria é constituída por especialistas que não exercem tais habilidades e quando as executam, fazem de forma fragmentada, com base no paradigma flexneriano, ou seja, no modelo curativo ${ }^{9}$.

O número de ESFs no Brasil vem aumentando e atualmente ainda há uma impactante diferença no número de equipes de Saúde da Família (SF) e equipes de Saúde Bucal (SB) cadastradas, o que sinaliza, tendo em vista as portarias ministeriais ${ }^{4,10,11}$, a oportunidade de criação de novas vagas para o cargo de CD: em julho de 2016, enquanto havia no Brasil 40.766 equipes de SF implantadas em 5.468 municípios brasileiros, a SB estava implantada em 5.024 municípios, os quais apresentavam 24.687 equipes.

Neste contexto de possibilidade de ampliação do mercado de trabalho e de necessidade de contar-se com a adequação do profissional ao perfil desejado, faz-se importante avaliar as características dos concursos públicos para contratação de CDs na ESF de municípios Brasileiros.

\section{MATERIAL E MÉTODOS}

Trata-se de pesquisa que utiliza dados secundários e públicos, dispensando avaliação por Comitê de Ética. A coleta dos dados foi realizada diretamente nos editais de abertura de concursos públicos para o cargo de CD na ESF no período de janeiro de 2006 a dezembro de 2014

Os dados foram coletados em duas bases de dados de grande circulação nacional: Odonto concursos (www.odontoconcursos.com.br) e ABITEP concursos (www.abitep.com.br). As variáveis analisadas foram: a) ano de realização 
do concurso; b) estado/região; c) conteúdo a ser abordado nas provas do concurso; d) avaliação de títulos; e) salário; f) número de vagas oferecidas; e g) regime de trabalho.

Após tabulação dos dados, realizou-se a análise descritiva por meio de tabelas de distribuição de frequência absoluta e relativa.

\section{RESULTADOS}

A tabela 1 apresenta a distribuição dos concursos e número de vagas oferecidas por macrorregião e ano de realização. O ano de 2007 apresentou o maior e 2010 o menor número de vagas oferecidas. $\mathrm{O}$ número médio de vagas disponíveis foi de 4,2, sendo a região Sudeste a que apresentou o maior número de vagas e concursos.

Neste período foram encontrados 765 editais. Dentre os estados, os que apresentaram maior número de editais foram Minas Gerais $(23,9 \%)$, Bahia $(5,5 \%)$, Santa Catarina $(9,9 \%)$, Mato Grosso (3,1\%) e Pará $(1,7 \%)$ (tabela 2).

$\mathrm{O}$ piso salarial médio oferecido foi de $\mathrm{R} \$$ 2.458,01 para 40 horas semanais de trabalho. A região Sul ofereceu o maior valor, $\mathrm{R} \$ 2.915,00$, seguida pelas regiões Sudeste e Centro-Oeste. As regiões Norte e Nordeste apresentaram menor piso salarial, com valor médio de $\mathrm{R} \$ 2.210,94 \mathrm{e}$ $\mathrm{R} \$ 2.114,42$, respectivamente (tabela 3 ).

Observou-se ainda variadas modalidades de contratação. A maioria dos editais (57\%) não apontou a modalidade de regime de regime de trabalho e o regime estatutário (21\%) prevaleceu sobre o regime celetista $(16 \%)$. Na região Centro-Oeste não foi observado nenhum edital adotando o regime de trabalho celetista, e na região Sul a frequência de regime CLT foi maior que a de regime estatutário (tabela 4).

Tabela 1 - Distribuição do número de vagas oferecidas no período de 2006 a 2014, por macrorregião

\begin{tabular}{|c|c|c|c|c|c|c|c|c|c|c|c|c|}
\hline \multirow{3}{*}{ Ano } & \multicolumn{10}{|c|}{ Macrorregião } & \multirow{2}{*}{\multicolumn{2}{|c|}{ Brasil }} \\
\hline & \multicolumn{2}{|c|}{ Norte } & \multicolumn{2}{|c|}{ Nordeste } & \multicolumn{2}{|c|}{$\begin{array}{l}\text { Centro- } \\
\text { Oeste }\end{array}$} & \multicolumn{2}{|c|}{ Sul } & \multicolumn{2}{|c|}{ Sudeste } & & \\
\hline & $\begin{array}{l}\tilde{o} \\
\tilde{\Xi} \\
\overline{0} \\
\tilde{0} \\
\tilde{U}\end{array}$ & $\begin{array}{l}\tilde{\pi} \\
\underset{\infty}{\infty} \\
>\end{array}$ & 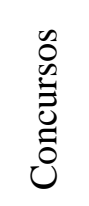 & 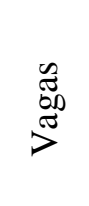 & 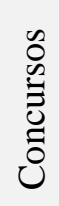 & 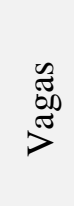 & $\begin{array}{l}0 \\
0 \\
0 \\
0 \\
0 \\
0\end{array}$ & 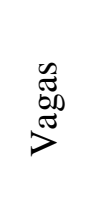 & $\begin{array}{l}0 \\
0 \\
\vdots \\
0 \\
0\end{array}$ & 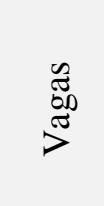 & $\begin{array}{l}0 \\
0 \\
\vdots \\
0 \\
0\end{array}$ & 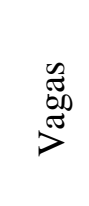 \\
\hline 2006 & 6 & 15 & 39 & 245 & 6 & 12 & 18 & 41 & 25 & 126 & 94 & 439 \\
\hline 2007 & 11 & 68 & 88 & 260 & 21 & 72 & 44 & 153 & 57 & 226 & 221 & 779 \\
\hline 2008 & 2 & 39 & 16 & 55 & 7 & 19 & 31 & 79 & 51 & 448 & 107 & 640 \\
\hline 2009 & 5 & 11 & 11 & 73 & 4 & 22 & 14 & 44 & 19 & 63 & 53 & 213 \\
\hline 2010 & 0 & 0 & 0 & 0 & 0 & 0 & 1 & 28 & 23 & 156 & 24 & 184 \\
\hline 2011 & 4 & 37 & 8 & 212 & 6 & 13 & 26 & 51 & 19 & 53 & 63 & 366 \\
\hline 2012 & 0 & 0 & 1 & 1 & 0 & 0 & 2 & 7 & 78 & 229 & 81 & 237 \\
\hline 2013 & 0 & 0 & 0 & 0 & 0 & 0 & 0 & 0 & 32 & 97 & 32 & 97 \\
\hline 2014 & 7 & 11 & 20 & 87 & 10 & 16 & 35 & 61 & 18 & 49 & 90 & 224 \\
\hline Total & 35 & 181 & 183 & 933 & 54 & 154 & 171 & 464 & 322 & 1447 & 765 & 3179 \\
\hline
\end{tabular}


Tabela 2 - Distribuição dos concursos realizados por macrorregião e estado

\begin{tabular}{|c|c|c|c|}
\hline Macrorregião & Estado & $\begin{array}{l}\text { Frequência absoluta } \\
\text { (n) }\end{array}$ & $\begin{array}{c}\text { Frequência relativa } \\
(\%)\end{array}$ \\
\hline \multirow{4}{*}{ Sudeste } & Minas Gerais & 183 & 23,9 \\
\hline & São Paulo & 98 & 12,8 \\
\hline & Rio de Janeiro & 25 & 3,3 \\
\hline & Espírito Santo & 15 & 2 \\
\hline \multicolumn{2}{|c|}{ Total parcial da região Sudeste } & 321 & 42 \\
\hline \multirow[t]{9}{*}{ Nordeste } & Bahia & 42 & 5,5 \\
\hline & Ceará & 31 & 4,1 \\
\hline & Sergipe & 26 & 3,4 \\
\hline & Paraíba & 24 & 3,1 \\
\hline & Alagoas & 18 & 2,4 \\
\hline & Pernambuco & 13 & 1,7 \\
\hline & $\begin{array}{l}\text { Rio Grande do } \\
\text { Norte }\end{array}$ & 12 & 1,6 \\
\hline & Piauí & 12 & 1,6 \\
\hline & Maranhão & 6 & 0,8 \\
\hline \multicolumn{2}{|c|}{ Total parcial da região Nordeste } & 184 & 24,1 \\
\hline \multirow[t]{3}{*}{ Sul } & Santa Catarina & 76 & 9,9 \\
\hline & Paraná & 51 & 6,7 \\
\hline & Rio Grande do Sul & 46 & 6 \\
\hline \multicolumn{2}{|c|}{ Total parcial da região Sul } & 173 & 22,6 \\
\hline \multirow[t]{4}{*}{ Centro-Oeste } & Mato Grosso & 24 & 3,1 \\
\hline & Goiás & 16 & 2,1 \\
\hline & Mato Grosso do Sul & 14 & 1,8 \\
\hline & Tocantins & 7 & 0,9 \\
\hline \multicolumn{2}{|c|}{ Total parcial da região Centro-Oeste } & 61 & 8 \\
\hline \multirow[t]{5}{*}{ Norte } & Pará & 13 & 1,7 \\
\hline & Rondônia & 6 & 0,8 \\
\hline & Acre & 3 & 0,4 \\
\hline & Amazonas & 3 & 0,4 \\
\hline & Roraima & 1 & 0,1 \\
\hline \multicolumn{2}{|c|}{ Total parcial da região Norte } & 26 & 3,4 \\
\hline \multicolumn{2}{|l|}{ Total geral } & 765 & 100 \\
\hline
\end{tabular}


Tabela 3 - Valor salarial oferecido, em Reais, nos editais avaliados

\begin{tabular}{|c|c|c|c|c|}
\hline Macrorregião & Média salarial (dp) & Mediana & Valor Máximo & Valor Mínimo \\
\hline Sul & $2.915,00( \pm 1.297,42)$ & $2.700,00$ & $8.092,81$ & 826,88 \\
\hline Sudeste & $2.451,80( \pm 1.322,44)$ & $2.198,00$ & $5.650,52$ & 502,32 \\
\hline Centro-oeste & $2.430,88( \pm 1.223,30)$ & $2.196,00$ & $5.943,78$ & $1.100,00$ \\
\hline Norte & $2.210,94( \pm 1.215,25)$ & $2.100,00$ & $4.500,00$ & 960,00 \\
\hline Nordeste & $2.114,42( \pm 1.799,11)$ & $2.000,44$ & $12.000,00$ & 415,00 \\
\hline Brasil & $2.458,01( \pm 1.138,51)$ & $2.231,62$ & $12.000,00$ & $\mathbf{4 1 5}, 00$ \\
\hline
\end{tabular}

Tabela 4 - Distribuição da amostra referente à modalidade de contrato de trabalho

\section{Modalidade de contrato de trabalho}

\begin{tabular}{lccccc}
\cline { 2 - 4 } Região & CLT & EST & PS/CT & CT & N/D \\
& $\mathrm{n}(\%)$ & $\mathrm{n}(\%)$ & $\mathrm{n}(\%)$ & $\mathrm{n}(\%)$ & $\mathrm{n}(\%)$ \\
\hline Sudeste & $71(22)$ & $77(24)$ & $22(7)$ & $6(2)$ & $144(45)$ \\
Nordeste & $13(7)$ & $26(14)$ & $2(1)$ & - & $144(78)$ \\
Sul & $36(21)$ & $33(19)$ & $7(4)$ & $5(3)$ & $92(53)$ \\
Centro-Oeste & - & $21(35)$ & - & - & $40(65)$ \\
Norte & $2(6)$ & $5(20)$ & - & - & $19(74)$ \\
BRASIL & $122(16)$ & $162(21)$ & $31(4)$ & $11(1)$ & $439(57)$ \\
\hline
\end{tabular}

CLT: celetista; EST: estatutário; PS/CT: processo seletivo para contrato temporário; CT: contrato temporário sem processo seletivo; N/D: não divulgado.

Os conteúdos que seriam exigidos não portuguesa (71\%). Apenas $14 \%$ exigiram foram divulgados em $15 \%$ dos editais. $\mathrm{Na}$ conhecimentos em saúde coletiva. Em $27 \%$ dos maioria deles exigiu-se conhecimentos editais foi levada em consideração a análise de específicos da área de atuação (81\%) e língua titulação (tabela 5). 
Tabela 5 - Distribuição dos editais em função dos conteúdos exigidos

\begin{tabular}{lcccccc}
\hline \multirow{2}{*}{ Conteúdos } & \multicolumn{2}{c}{ Exigido } & \multicolumn{2}{c}{ Não exigido } & \multicolumn{2}{c}{ Sem divulgação } \\
\cline { 2 - 7 } & $\mathrm{n}$ & $\%$ & $\mathrm{n}$ & $\%$ & $\mathrm{n}$ & $\%$ \\
\hline Conhecimentos Específicos & 618 & 81 & 29 & 04 & 118 & 15 \\
Língua Portuguesa & 538 & 71 & 109 & 14 & 118 & 15 \\
Títulos* & 208 & 27 & 439 & 58 & 118 & 15 \\
Conteúdos Diversos (outros)** & 250 & 33 & 397 & 52 & 118 & 15 \\
Conhecimentos Gerais & 225 & 30 & 422 & 55 & 118 & 15 \\
Conhecimentos em Saúde Coletiva & 108 & 14 & 539 & 71 & 118 & 15 \\
\hline Doutorado, Mestrado, Especialização, Atualização, Tempo de serviço público & & & \\
** Matemática, raciocínio lógico, informática & \multicolumn{7}{c}{. } \\
\hline
\end{tabular}

\section{DISCUSSÃO}

Para a contratação de profissional efetivo toda a Administração Pública deve submeter os candidatos ao concurso de provas ou de provas e títulos conforme prescreve o inciso II do art. 37 da Constituição da República ${ }^{12}$.

A Portaria $n^{\circ}$ 648/GM de 28 de março de 2006 aprovou a Política Nacional de Atenção Básica, estabelecendo normas para a organização da Atenção Básica para o PSF ${ }^{10}$. Assim, avaliar editais dos concursos públicos a partir deste ano torna-se importante, partindo-se do princípio de que as solicitações de concursos devem ser fundamentadas de acordo com o perfil profissional para a atuação no serviço público de saúde, atendendo às necessidades dos usuários de forma resolutiva e integral.

Avaliando as regiões brasileiras observouse um maior número de concursos na região Sudeste seguido pela Nordeste com uma média de 4,1 vagas por região. Considerando a lógica demográfica, a região Nordeste apresenta 9 Estados e possui cerca de 1794 municípios, seguido da região Sudeste que apresenta 4 Estados e 1668 municípios, a região Sul ocupa a terceira posição apresentando 3 Estados e 1191 municípios. As regiões Centro-Oeste e Norte ocupam as últimas posições com 3 Estados e 1 Distrito Federal e 466 municípios e 7 Estados e 450 municípios, respectivamente ${ }^{13}$. Assim, o número maior de municípios por macrorregião justificaria o maior número de concursos realizado na região Sudeste e Nordeste, no período de avaliação.

Avaliando anualmente observou-se um maior número de editais no ano de 2007, o que pode ser justificado pela publicação, em 2006, da portaria $822^{14}$ que estabeleceu os repasses financeiros para a ESF e, pouco depois, com a publicação das portarias $90 \mathrm{GM} / \mathrm{MS}^{15}$ de 17 de janeiro de 2008 e 2920 GM/MS de 3 de dezembro de $2008^{16}$ que reforçam o incentivo financeiro. Além disso, o que poderia justificar a maior frequência de concursos até o ano de 2009 é a publicação de legislações: Portaria $2371 \mathrm{GM} / \mathrm{MS}^{17}$ que instituiu as unidades odontológicas móveis e a Portaria 2372GM/MS ${ }^{18}$ que criou o plano de fornecimento de equipamentos odontológicos para as equipes de saúde, o que motivou os municípios implantarem equipes de SB na ESF.

Considerando a análise da média salarial, percebe-se que as regiões Sul e Sudeste, além de terem a maior concentração de CDs, são também 
aquelas que apresentam melhor desenvolvimento econômico $^{19}$ e, consequentemente, oferecem melhor piso salarial. No entanto, a média salarial observada ficou aquém da preconizada na legislação vigente ${ }^{20}$ : até três salários mínimos para carga horária de 20 horas semanais para Médicos e CDs, acrescida de $40 \%$ referente ao adicional de insalubridade.

Isso pode acarretar uma migração de profissionais de um município para o outro em busca de melhores salários, o que gera problema no gerenciamento de recursos humanos na Atenção Básica. As ações em saúde neste nível de atenção se caracterizam predominantemente pelas relações interpessoais que se estabelecem de forma longitudinal, daí a importância que assume a estabilidade e o vínculo de trabalho dos profissionais das equipes de saúde.

A forma de contratação também se relaciona com o vínculo profissional. Observouse que a contratação pelo regime estatutário prevaleceu de forma geral no Brasil e o regime celetista prevaleceu nas regiões Sul e Sudeste. O vínculo celetista não apresenta o direito à estabilidade prevista para estatutários, levando ao não fortalecimento do vínculo com a comunidade pela grande rotatividade de profissionais que buscam por estabilidade no emprego $^{21,22}$.

Com relação aos domínios exigidos, apenas $14 \%$ cobraram conhecimentos relacionados à Saúde Pública/Coletiva. É primordial que haja profissionais de saúde dotados de uma visão mais humanística voltados para prestar cuidados contínuos e resolutivos à comunidade. Assim os editais de concursos, que representam a forma de seleção destes profissionais para o trabalho na ESF, deveriam exigir estes conteúdos em suas provas de seleção, permitindo o alinhamento entre o perfil do profissional e as exigências do sistema de saúde.

Para trabalhar no SUS com qualidade e atender às necessidades da população, é necessário ser um profissional generalista, tecnicamente competente e com sensibilidade social. No entanto, para que isto seja de fato efetivado, é preciso que haja articulação entre ensino superior e saúde, fundamental no âmbito da ESF, com polos de Capacitação, Formação e Educação Permanente de pessoal para a SF.

Notou-se importante discrepância quanto à conformação dos editais para seleção CDs da ESF, pois quanto ao conteúdo programático abordado foi observado que este difere muito de um município para o outro. A maioria privilegia as questões de conhecimentos específicos (técnicos) em detrimento aos conhecimentos de Saúde Coletiva, não contemplando habilidades e competências necessárias para atuação nas ESFs.

A ausência de uma lei de concursos que deveria ser editada não pela União, mas por cada um dos entes federados, em que pese poder significar algum grau de prejuízo na condução dos referidos certames, porque eliminaria discussões que não raramente batem às portas do Poder Judiciário, não impede que se estabeleçam as exigências para o provimento de cargos e empregos nas leis responsáveis pela sua criação $^{24}$. No entanto, numa perspectiva mais ampla espera-se que seja implementada uma Política Ministerial visando exames mais apurados na seleção dos CDs, tendo em vista todas as prerrogativas descritas nesta pesquisa e nas legislações de SB vigentes e que esta pesquisa subsidie novas investigações que possam olhar para as diferentes vertentes da gestão de recursos humanos, especialmente no que compete à alta rotatividade de profissionais no âmbito das equipes de SB da ESF.

\section{CONCLUSÃO}

Os concursos públicos realizados no Brasil, no período avaliado, mostraram editais imprecisos, com ausência de informações importantes para a decisão segura em participar do processo seletivo. A maioria dos concursos foi 
realizada na região Sudeste, estado de Minas Gerais. Na maioria exigiu-se conhecimentos específicos da área de atuação e língua portuguesa. Conteúdos de Saúde Coletiva foram exigidos apenas pela minoria. Faz-se necessário o direcionamento, principalmente em relação aos conhecimentos exigidos nas provas, para que seja contemplado o profissional com perfil e conhecimentos adequados para o trabalho na ESF.

\section{ABSTRACT \\ Characteristics of exams for hiring dentists for the Family Health Strategy}

The aim of the present study was to evaluate the characteristics of public exams for hiring dental surgeons for the Family Health Strategy in Brazilian municipalities. Documentation of public tenders issued in Brazil between 2006 and 2014 was analyzed, based on the following criteria: macro region, programmatic content, salary, number of vacancies, and hiring system. Of a total number if 765 tenders for applications to do the exam, $42 \%$ were for the Southeastern region; $24 \%$ for the Northeastern; $22 \%$ for the Southern; $7 \%$ for the Midwestern, and 5\% for the Northern region. The mean number of vacancies offered was 4.2 , and the mean salary was $\mathrm{R} \$$ 2.458,01 (Brazilian Reais). The contents of the exams were distributed into the categories of specific knowledge (81\%), Portuguese language (71\%), general knowledge $(30 \%)$, collective health $(14 \%)$ and others $(33 \%)$. Relative to the forms of hiring, $21 \%$ were related to the statutory work regime, $16 \%$ were contracted under the laws for employees which govern the private sector (consolidated labor law), 4\% selection process of a temporary nature, $1 \%$ temporary hiring in the absence of the public selection process and $57 \%$ did not specify the type of contract. The public exams in the period evaluated showed imprecise tender documents, with the absence of important information for making a secure decision to participate in the selection process. The majority of the exams were held in the Southeastern region, and state of Minas Gerais, with the demand for specific knowledge of the area of work and Portuguese language. Contents relative to Collective Health and public titles were demanded only by the minority of tenders. It is necessary for tenders to be more specific, particularly with regard to the knowledge demanded in the exams, so that the professional with the adequate profile and knowledge will be hired to work in the Family Health Strategy.

Descriptors: Dentists. Personnel Selection. Unified Health System. Family Health.

\section{REFERÊNCIAS}

1. Unfer B. O Cirurgião-Dentista do Serviço Público: Formação, Pensamento e Ação. Rev Bras Odontol Saúde Coletiva. 2001;2(2):45-51.

2. Viana ALD, Dal Poz MR. A Reforma do Sistema de Saúde no Brasil e o Programa de Saúde da Família. Physis Rev Saúde Coletiva. 2005;12(supl):S255-64.

3. Nascimento APS, Santos LF, Carnut L. Atenção primária à saúde via estratégia saúde da família no Sistema Único de Saúde: uma introdução sobre os problemas inerentes à operacionalização de suas ações. J Manag Prim Health Care. 2011;2(1):18-24.

4. Brasil. Ministério da Saúde. Portaria n. 267/GM, de 06 março de 2001. Define as Diretrizes e Normas da inclusão das ações de saúde bucal no PSF. Diário Oficial da República Federativa do Brasil, Brasília (DF); 2001 mar 7; Seção 1:67. [Acesso em 2016 fev. 15]. Disponível em https:// www.nescon.medicina.ufmg.br/biblioteca/.

5. Silveira Filho AD. A Saúde Bucal no PSF: o desafio de mudar a prática. Rev Saúde Família. 2002 dez, 6:36-43

6. Brasil. II Conferência Nacional de Saúde Bucal. Brasília, DF: Ministério da Saúde; 1993.

7. Valença AMG. A Educação em Saúde na Formação do Cirurgião-Dentista: da necessidade à prática participativa. Niterói: 
Editora UFF; 1998.

8. Brasil. Conselho Nacional de Educação. Câmara de Educação Superior Resolução CNE/CES 3. Institui Diretrizes Curriculares Nacionais do Curso de Graduação em Odontologia. 2002 [Acesso em 3 jan. 2016]. Disponível em: http://portal.mec.gov.br/cne/ arquivos/pdf/CES032002.pdf.

9. Rodrigues MP, Araújo MSSA. O processo de trabalho em saúde da família. 2008 [Acesso em 15 fev. 2016]. Disponível em: http://www.observatório.nesc.ufrn.br.

10. Brasil. Ministério da Saúde. Portaria 648/GM, de 28 de março de 2006. Aprova a Política Nacional de Atenção Básica, estabelecendo a revisão de diretrizes e normas para a organização da Atenção Básica para o Programa Saúde da Família (PSF) e o Programa Agentes Comunitários de Saúde (PACS). Diário Oficial da República Federativa do Brasil, Brasília (DF); 2006.

11. Brasil. Ministério da Saúde. Portaria $n^{\circ}$ 2.488, de 21 de outubro de 2011. Aprova a Política Nacional de Atenção Básica, estabelecendo a revisão de diretrizes e normas para a organização da Atenção Básica, para a Estratégia Saúde da Família (ESF) e o Programa de Agentes Comunitários de Saúde (PACS). Diário Oficial da República Federativa do Brasil, Brasília (DF); 201. [Acesso em 3 jan. 2016]. Disponível em: http://bvsms.saude.gov.br /bvs/saudelegis/gm/2011/prt2488_21_10_2 011.html.

12. Brasil. Presidência da República. Casa Civil. Subchefia para Assuntos Jurídicos. Constituição da República Federativa do Brasil de 1988. [Acesso em 5 mai. 2016]. Disponível em: http://www.planalto.gov. br/ccivil_03/Constituicao/Constituicao.htm.

13. IBGE. Instituto Brasileiro de Geografia e Estatística (2009). Perfil dos Municípios
Brasileiros - 2009. Arquivado desde o original em 21 de janeiro de 2013. [Acesso em 5 mai. 2016]. Disponível em: http:// www.ibge.gov.br/home/estatistica/economi a/perfilmunic/2009/.

14. Brasil. Ministério da Saúde. Portaria $n^{\circ} 822$, de 17 de abril de 2006. Altera critérios para definição de modalidades das ESF dispostos na Política Nacional de Atenção. [Acesso em 5 mai 2016]. Disponível em: http:// bvsms.saude.gov.br/bvs/saudelegis/gm/200 6/prt0822_17_04_2006.html.

15. Brasil. Ministério da Saúde. Portaria $\mathrm{n}^{\circ}$ 90, de 17 de janeiro de 2008. Atualiza o quantitativo populacional de residentes em assentamentos da reforma agrária e de remanescentes de quilombos, por município, para cálculo do teto de Equipes Saúde da Família, modalidade I, e de Equipes de Saúde Bucal da estratégia Saúde da Família. [Acesso em 5 mai. 2016]. Disponível em: http://bvsms.saude.gov.br/bvs/saudelegis/g m/2008/prt0090_17_01_2008.html.

16. Brasil. Ministério da Saúde. Portaria $n^{\circ}$ 2.920, de 2 de dezembro de 2008. Estabelece recursos financeiros para Municípios com equipes de Saúde da Família que atuem em áreas priorizadas para o Programa Nacional de Segurança Pública com Cidadania. [Acesso em 5 mai. 2016]. Disponível em: http://bvsms.saude.gov.br/bvs/saudelegis/g m/2008/prt2920_02_12_2008.html.

17. Brasil. Ministério da Saúde. Portaria $n^{\circ}$ 2.371, de 7 de outubro de 2009. Institui, no âmbito da Política Nacional de Atenção Básica, o Componente Móvel da Atenção à Saúde Bucal - Unidade Odontológica Móvel - UOM. [Acesso em 5 mai. 2016]. Disponível em: http://bvsms.saude.gov.br/ bvs/saudelegis/gm/2009/prt2371_07_10_20 09.html.

18. Brasil. Ministério da Saúde. Portaria $n^{\circ}$ 2.372, de 7 de outubro de 2009. Cria o plano 
de fornecimento de equipamentos odontológicos para as Equipes de Saúde Bucal na Estratégia Saúde da Família. [Acesso em 5 mai. 2016]. Disponível em: http://bvsms.saude.gov.br/bvs/saudelegis/g m/2009/prt2372_07_10_2009.html.

19. Frydman M. Boletim Informativo: veja o número de Faculdades de Odontologia no Brasil [online] São Paulo: Odontoconcursos; 2015. [Acesso em 10 jan. 2015]. Disponível em: http://www.odontoconcursos.com.br/ boletim.

20. Brasil. Lei $\mathrm{n}^{0} 3.999$, de 15 de dezembro de 1961. Presidência da República. Casa Civil. Subchefia para Assuntos Jurídicos. Altera o salário-mínimo dos médicos e cirurgiões dentistas. [Acesso em 5 mai. 2016]. Disponível em: http://www.planalto.gov.br/ ccivil_03/leis/1950-1969/L3999.htm.

21. Magnago C, Pierantoni CR. Cenário de contratações para a Estratégia Saúde da
Família no município do Rio de Janeiro: análise dos anos 2000. J Manag Prim Health Care. 2013;4(2):116-25

22. Facó EF, Viana LMO, Bastos VA, Nuto SAS. O Cirurgião-Dentista e o programa saúde da família na microrregião II, Ceará, Brasil. Rev Bras Promoç Saúde. 2005;18(2): 70-7.

23. Morita MC, Kriger L. Mudanças nos cursos de Odontologia e a interação com o SUS. Rev ABENO. 2004;4(1):17-21.

24. Fortini C, Vieira VK. Ponderações sobre a prova de títulos nos concursos públicos. Revista do Tribunal de Contas do Estado de Minas Gerais, Edição Especial, ano XXVIII, 2010.

Correspondência para:

Profa. Flávia Martão Flório

e-mail: flavia.florio@ slmandic.edu.br

Rua José Rocha Junqueira, 13

13045-755 - Ponte Preta - Campinas/SP 